\title{
Condiciones laborales, riesgo y salud de los trabajadores forestales de Misiones, Corrientes y Entre Ríos (Argentina), 2010-2014
}

\author{
Labor conditions, risk and health of forestry workers \\ in Misiones, Corrientes and Entre Ríos (Argentina), \\ 2010-2014
}

'Licenciada en Geografía, magíster en Ciencias Sociales. Profesional Principal, Centro de Estudios e Investigaciones Laborales, Consejo Nacional de Investigaciones Científicas y Técnicas (CEIL-CONICET), Ciudad Autónoma de Buenos Aires, Argentina. $\triangle$ iD

'Licenciada en Sociología, doctora en Geografía. Investigadora Adjunta, Centro de Estudios e Investigaciones Laborales, Consejo Nacional de Investigaciones Científicas y Técnicas (CEIL-CONICET), Ciudad Autónoma de Buenos Aires, Argentina. $\bowtie$ iD
RESUMEN En la Argentina, en las últimas décadas, la actividad forestal ha evidenciado una importante expansión promovida por el Estado a través de incentivos que alentaron la implantación de especies forestales. En este artículo se examinan las condiciones laborales de un número de trabajadores forestales de las tres principales provincias productoras de madera de la Argentina (Corrientes, Misiones y Entre Ríos). Con los datos de la Encuesta sobre Empleo, Protección Social y Condiciones de Trabajo de los Asalariados Agrarios se efectuó un procesamiento y análisis de la información referida a los trabajadores forestales de las provincias mencionadas $(n=113)$. A su vez, la realización de entrevistas en profundidad a trabajadores $(n=30)$ y contratistas forestales $(n=8)$, entre 2010 y 2014, contribuyó a explicar el fenómeno estudiado en su totalidad. Los resultados obtenidos muestran que más del $50 \%$ de los trabajadores considera estar expuesto a condiciones desfavorables del medio ambiente físico de trabajo. El 16\% ha tenido un accidente laboral en el último año. La alta precariedad de este tipo de empleo, sumada a las formas de contratación (tercerización), dificulta la identificación de acciones a seguir para revertir los procesos analizados. PALABRAS CLAVES Condiciones de Trabajo; Salud Laboral; Forestación; Argentina.

\begin{abstract}
In the last decades in Argentina, forestry activity has seen an important expansion, promoted by the State through incentives encouraging the implantation of forest species. This article examines the working conditions of a number of forestry workers in the three main timber producing provinces of Argentina (Corrientes, Misiones and Entre Ríos). Using data from the Survey on Employment, Social Protection and Labor Conditions of Salaried Agricultural Workers, information on forest workers of the aforementioned provinces was processed and analyzed $(n=113)$. In addition, indepth interviews with workers $(n=30)$ and forest contractors $(n=8)$, carried out from 2010-2014, contributed to the explanation of the studied phenomenon as a whole. The results show that more than $50 \%$ of workers consider themselves exposed to unfavorable conditions in the physical working environment, with $16 \%$ having had a work accident in the last year. The high precariousness of this type of employment, in conjunction with the forms of contracting (outsourcing), makes it difficult to identify actions to be taken to reverse the processes analyzed.
\end{abstract}

KEY WORDS Working Conditions; Occupational Health; Forestry; Argentina. 


\section{INTRODUCCIÓN}

La actividad forestal es considerada mundialmente una de las más riesgosa. Una extensa bibliografía da cuenta de las malas condiciones laborales a las que se ve expuesto el trabajador y las consecuencias que esto tiene sobre su salud. La Organización Internacional del Trabajo observa que -junto con la construcción y la minería- es de los sectores más riesgosos por la alta incidencia de accidentes y de siniestros mortales en el desempeño laboral ${ }^{(1)}$.

Las distintas tareas exigen un gran compromiso del cuerpo $y$, a su vez, una alta exposición a condiciones físicas adversas. Por otra parte, el trabajador suele vivir en las plantaciones donde se instalan los campamentos, por lo general, lugares aislados y mal comunicados con los centros urbanos, lo que suele dificultar el acceso a los centros médicos o la asistencia inmediata cuando ocurre una emergencia. Otras cuestiones importantes que hacen a la calidad de vida del trabajador forestal durante la permanencia en el obraje, y que muchas veces no reúnen las condiciones necesarias, son la habitacional y el suministro de alimentos y de agua potable. La dispersión y el aislamiento en las plantaciones, lejos de los centros poblados, y la gran movilidad de los obreros forestales colaboran con su invisibilidad, a lo que se agrega la carencia de estadísticas que reflejen la dimensión de la mano de obra involucrada. Por otra parte, la intensidad del trabajo, las condiciones y el medio ambiente en los que se lleva a cabo hacen que, en distintos países productores de madera, la mano de obra ocupada sea mayoritariamente masculina.

La actividad forestal en nuestro país ha evidenciado una importante expansión, promovida por el Estado a través de incentivos que alentaron la implantación de especies forestales. Las provincias de Misiones, Corrientes y Entre Ríos son las responsables de más del $90 \%$ de la producción de madera de la Argentina, con una superficie implantada de 932.379 hectáreas $(77,36 \%$ del total nacional) $^{(2,3)}$ (Tabla 1). La producción maderera

Tabla 1. Superficie total forestada (en hectáreas) y producción total (en toneladas), por especie y provincia. Argentina, 2013.

\begin{tabular}{|c|c|c|c|c|}
\hline \multirow[t]{2}{*}{ Provincias y especies } & \multicolumn{2}{|c|}{ Superficie (en hectáreas) } & \multicolumn{2}{|c|}{ Producción (en toneladas) } \\
\hline & $\mathrm{n}$ & $\%$ del total país & $\mathrm{n}$ & $\%$ del total país \\
\hline \multicolumn{5}{|l|}{ Corrientes } \\
\hline Coniferas & 312.369 & - & 2.337.287 & - \\
\hline Eucalipto & 107.457 & - & 1.596 .522 & - \\
\hline Otras & 6.160 & - & - & - \\
\hline Total provincial & 425.987 & 35,3 & 3.933 .809 & 34,5 \\
\hline \multicolumn{5}{|l|}{ Entre Ríos } \\
\hline Coníferas & 20.174 & - & 159.325 & - \\
\hline Eucalipto & 106.281 & - & 1.575 .587 & - \\
\hline Salicáceas & 26.967 & - & 10.623 & - \\
\hline Otras & 577 & - & - & - \\
\hline Total provincial & 153.999 & 12,8 & 1.745 .535 & 15,3 \\
\hline \multicolumn{5}{|l|}{ Misiones } \\
\hline Coniferas & 306.592 & - & 4418.944 & - \\
\hline Eucalipto & 10.557 & - & 170.666 & - \\
\hline Otras & 35.243 & - & 57.756 & - \\
\hline Total provincial & 352.392 & 29,2 & 4.647.366 & 40,77 \\
\hline Total de las tres provincias & 932.379 & 77,4 & 10.326 .710 & 90,6 \\
\hline Total país & 1.205 .317 & 100,0 & 11.399 .225 & 100,0 \\
\hline
\end{tabular}


está destinada a la elaboración de celulosa y papel, la fabricación de tableros de fibra de densidad media, la producción de madera sólida y el aserrado.

La extracción de la madera se encuentra tercerizada a través de contratistas de servicios, como ocurre en la mayoría de los países con actividad forestal ${ }^{(4,5,6)}$. Las ocupaciones se relacionan con diferentes tareas: plantación, control de malezas y plagas, raleo, volteo, apeo, carga y transporte de la madera. La mecanización de la cosecha se encuentra más difundida en la provincia de Misiones, donde grandes empresas disponen de extensas superficies plantadas que justifican la inversión en equipos, en tanto en las otras dos provincias son pocos los establecimientos que disponen de cosechadoras. Se estima que, en 2005, 4.391 trabajadores estaban ocupados directamente en el sector primario forestal de la Mesopotamia ${ }^{(7)}$.

En el periodo 2002-2004, la extracción de la madera encabezaba el ranking de incidencia de accidentes y de enfermedades profesionales por actividades en la Argentina (índice de incidencia de 195/1.000 trabajadores), poniendo de relieve las condiciones y los peligros de esta ocupación ${ }^{(7)}$.

La subcontratación de trabajadores por parte de prestadores de servicios ha generado una mayor rotación de la mano de obra, dificultando en buena medida su calificación. Muchas veces el "saber hacer" del trabajador es adquirido exclusivamente con la experiencia, sin que en el aprendizaje de la tarea medien instancias de capacitación ${ }^{(8)}$. Esta informalidad en el aprendizaje, a la que se agregan el empleo de métodos inadecuados en la tala de árboles y en la extracción de la madera, así como la rotación laboral, podrían incidir en la alta siniestralidad de la actividad y en la presencia de patologías ${ }^{(9)}$.

Otra particularidad del trabajo forestal es el predomino de un sistema de remuneración a destajo o por rendimiento que alienta el ritmo de trabajo rápido y la autoexplotación de la mano de obra. La intensificación del ritmo produce un desgaste físico que impacta negativamente en la salud del trabajador y, además, conspira en contra de la adopción de buenas prácticas en el desarroIlo de la tarea, orientadas a prevenir accidentes y lesiones ${ }^{(6)}$. Más aún, esta modalidad de remuneración por productividad, al acelerar el ritmo de trabajo, puede colaborar con el aumento de accidentes ${ }^{(1)}$.

De esta manera, el contexto de la producción forestal donde se articulan mecanismos formales y no formales en la organización del proceso de trabajo y la atención a prácticas y normativas de prevención y control de la calidad ocupacional, reviste cierta complejidad. Es decir, junto al avance de la institucionalización de normativas conviven formas de relacionarse con el trabajo producto de los espacios de socialización y del entorno social de los trabajadores que, por no estar institucionalizadas, no dejan de ser efectivas en función de la salud de la mano de obra.

En este artículo se examinan las condiciones laborales de trabajadores forestales de las tres principales provincias madereras de la Argentina (Corrientes, Misiones y Entre Ríos), a partir del análisis de los resultados de una encuesta más amplia realizada a trabajadores agrarios y de entrevistas en profundidad a trabajadores y contratistas forestales.

Se evalúa la situación de la mano de obra forestal teniendo en cuenta la temporalidad de las ocupaciones (como manifestación de estabilidad-inestabilidad laboral), la formalización de la ocupación (disponibilidad de beneficios sociales: aportes jubilatorios, cobertura de salud, vacaciones, etcétera). Asimismo, el análisis de las condiciones laborales comprende la seguridad en el desempeño de la tarea, el uso de elementos de protección personal, el medio ambiente físico en el que se desenvuelve y los factores de riesgo a los que se expone (temperaturas extremas, ruido, vibraciones, contaminantes químicos), la duración de la jornada de trabajo, los momentos de descanso, entre otras. Se observan, también, cuáles son los problemas de salud ocasionados por el trabajo, la percepción que el trabajador tiene de su estado de salud, la ocurrencia y las causas de accidentes de trabajo en los últimos 12 meses y si dispone de cobertura por parte de una aseguradora de riesgos del trabajo (ART). Se releva además si 
el trabajador ha sido capacitado por su empleador en temas de prevención y seguridad, técnicas de trabajo y manejo de herramientas.

\section{Trabajo, salud y riesgos}

La relación trabajo, riesgos laborales, enfermedad y salud es una temática que ha sido abordada desde distintas perspectivas. Es así como determinados estudios se orientan a analizar el contexto en el que se desarrolla un trabajo y en cómo se organiza el proceso laboral, como forma de constitución del marco estructural de la salud ocupacional ${ }^{(10)}$. No obstante, cabe señalar que todas estas dimensiones de los mercados laborales no están dadas de una vez y para siempre, sino que son el resultado del momento histórico, del poder de negociación de las partes y de las particularidades de las geografías en las que se inscriben, por lo tanto, una de sus especificidades distintivas es su inherente dinámica.

Asimismo, la noción de salud ha ido cambiando a lo largo del tiempo, tanto desde la propia medicina (somática-fisiológica, psíquica, sanitaria), como desde concepciones sociales de la salud ideal (cultural, económica y político-legal) ${ }^{(11)}$. Inicialmente la salud era definida como la ausencia de enfermedad. En la década de 1960, la Organización Mundial de la Salud define la salud ideal como "...un estado de completo bienestar físico, mental y social", reconociendo la integración cuerpomente. Sin embargo, posteriormente, se comienza a prestar atención a factores que se vinculan con las creencias, los valores, las normas y los conocimientos en relación con la salud y con la enfermedad. Si bien la enfermedad es entendida como una dolencia del individuo, también es una construcción social y cultural, que puede comprender tanto un contexto familiar, como un entorno institucional y social más amplio ${ }^{(11)}$. En este sentido, Menéndez observa que las condiciones de vida y de trabajo son el emergente de condicionantes sociales y no un problema exclusivo del ámbito médico ${ }^{(12)}$.

Específicamente en el mundo del trabajo, la percepción del trabajador acerca del impacto negativo que el trabajo tiene sobre su salud es analizada por otros autores no solo con relación a condiciones "reales" u "objetivas", sino a ciertos atributos del individuo, como pueden ser la juventud, la capacidad de resistencia al esfuerzo físico y a soportar intensas jornadas de trabajo, así como a cuestiones culturales, que pueden llevar a que el trabajador, además de minimizar los riesgos, los tenga totalmente naturalizados, al punto de considerarlos inherentes al desarrollo de su trabajo ${ }^{(13)}$. La percepción y la propensión a tomar riesgos en el desempeño de una tarea a lo largo de la vida laboral, se relaciona con su pertenencia de clase, con construcciones socioculturales y, también, con atributos adjudicados al género ${ }^{(14)}$.

Por otra parte, desde distintas posiciones teóricas se ha intentado construir una noción del riesgo, en general, y del riesgo laboral, en particular, con foco en concepciones más objetivas (condiciones materiales), más subjetivas (vinculadas a cuestiones culturales, valores y creencias) o bien surgidas de la integración de ambas. Es así como, desde la necesidad de realizar un control del riesgo, algunas perspectivas ponen la mirada en los efectos adversos que producen ciertos comportamientos o prácticas inseguras y las inapropiadas condiciones del entorno laboral. La estimación del riesgo, a través del registro estadístico de las probabilidades de la ocurrencia de siniestros o de efectos negativos sobre la salud de los individuos, constituye muchas veces el fundamento del análisis unidireccional causa-efecto.

Por el contrario, la percepción del riesgo en función del contexto, de la pertenencia cultural y de clase, aparece como opuesta a una concepción objetivista y, también, racionalista ${ }^{(15)}$. Para esta autora, aquello que los individuos asumen como riesgo tiene más que ver con las ideas sociales de moral y justicia que ellos definen, que con cuestiones probabilísticas de costos y beneficios en la aceptación del riesgo. La familiaridad del contexto y el sistema de socialización en el cual se encuentra inserto determinado grupo social conducen al desarrollo de un sentido compartido de inmunidad subjetiva, es decir, 
el desarrollo de tendencias a minimizar resultados negativos frente al riesgo ${ }^{(15)}$. La integración de las relaciones de poder, los aspectos psicosociales de las relaciones y la pertenencia sociocultural de los sujetos son señalados como indispensables de integración desde la sociología del riesgo ${ }^{(16)}$.

En la actualidad, las actividades económicas ancladas en la relación capital-trabajo han estimulado la incorporación de elementos de prevención y uso que tiendan a minimizar los riesgos laborales en el marco de la protección social del trabajo. Para ello, se avanzó en mecanismos formales -legislaciones y controles- y en sistemas de estandarización de calidad por parte de las empresas. Se ha desarrollado una cultura preventiva y de seguridad que involucra valores y comportamientos del conjunto de la sociedad, de los colectivos y de organizaciones ocupadas en el mejoramiento de las condiciones laborales ${ }^{(17)}$.

En concordancia con lo anterior, las condiciones y el medio ambiente de trabajo están determinados por el proceso de trabajo y el contexto socioeconómico en el que se llevan a cabo las distintas tareas en relación con las exigencias, los requerimientos y las limitaciones del puesto de trabajo. El efecto negativo de las condiciones y el medio ambiente de trabajo puede percibirse no solo en las dimensiones físico-biológicas, sino también en factores psíquicos, mentales y sociales ${ }^{(18,13)}$, relacionados con el contenido y la organización del trabajo. En este sentido, las condiciones adversas en la salud del trabajador y los riesgos a los que se ve expuesto como efecto de factores físico-biológicos y psicosociales negativos, generan padecimientos, enfermedades profesionales e, incluso, pueden ser la causa de accidentes.

Se reconoce que el desempeño de un trabajo y las condiciones en las que este se realiza se vinculan directamente y en forma continua con la salud del individuo. En las distintas ocupaciones, empresas y organizaciones del proceso de trabajo se entablan diversas formas de relación con respecto a la producción, generándose comportamientos, hábitos y valores que involucran, entre otras cosas, a la prevención. Se puede hablar entonces de la construcción de una identidad socioprofesional de los colectivos dentro de una cultura específica de trabajo, en la que el riesgo puede ser considerado como un recurso identitario y que, además, esa cultura colabora en la construcción de la idea de salud que tienen esos trabajadores ${ }^{(19)}$.

A partir de lo anterior, este artículo busca analizar, en el contexto de la producción forestal, cómo se construye la implementación de las condiciones laborales entre el mundo del trabajo y el mundo empresarial, la incidencia específica actual, considerando este proceso mutuamente condicionado por valores, lógicas y estrategias que afianzan y, también, obstaculizan la generalización de estas prácticas.

\section{MATERIALES Y MÉTODOS}

La fuente de datos utilizada para el análisis cuantitativo es la Encuesta sobre Empleo, Protección Social y Condiciones de Trabajo de los Asalariados Agrarios, realizada por el Ministerio de Trabajo, Empleo y Seguridad Social de la Nación, conjuntamente con el Registro Nacional de Trabajadores y Empleadores Agrarios y la Superintendencia de Riesgos del Trabajo, cuyos datos fueron publicados en $2016^{(20)}$. Esta Encuesta fue aplicada en 10 provincias de la Argentina, durante 2013 y 2014, en hogares de áreas rurales (dispersas y concentradas) y urbanas de hasta 25.000 habitantes, con al menos un asalariado ocupado en actividades agrarias entre sus integrantes, durante los últimos 12 meses. El diseño de la muestra de los hogares se realizó con base en la selección de 80 segmentos censales (rurales y urbanos, del tamaño antes especificado), según el Censo Nacional de Población, Hogares y Vivienda de 2010. En cada segmento se seleccionaron aleatoriamente cinco viviendas con trabajadores agrarios para encuestar. Se realizaron 400 encuestas por provincia. Las secciones y las dimensiones del cuestionario de la Encuesta se pueden observar en la Tabla 2. 
Tabla 2. Secciones y dimensiones de la Encuesta sobre Empleo, Protección Social y Condiciones de Trabajo de los Asalariados Agrarios.

\begin{tabular}{|c|c|}
\hline Secciones & Dimensiones \\
\hline INFORMACIÓN BÁSICA DEL HOGAR & $\begin{array}{l}\text { Composición } \\
\text { Edad } \\
\text { Sexo } \\
\text { Nivel educativo }\end{array}$ \\
\hline INFORMACIÓN LABORAL & $\begin{array}{l}\text { Condición de actividad de los > } 14 \text { años } \\
\text { Tipo de ocupación } \\
\text { CICLO DE TRABAJO AGRARIO PARA LOS ASALARIADOS (MES A MES DEL AÑO DE REFERENCIA) } \\
\text { Ocupación } \\
\text { Tarea } \\
\text { Período trabajado } \\
\text { Producto agrario involucrado } \\
\text { Tipo de trabajo (permanente o transitorio) } \\
\text { Percepción de beneficios sociales } \\
\text { Formas de contratación } \\
\text { Presencia o no de migración }\end{array}$ \\
\hline $\begin{array}{l}\text { INFORMACIÓN SOBRE LAS } \\
\text { CONDICIONES DE TRABAJO DEL } \\
\text { ASALARIADO AGRARIO }\end{array}$ & $\begin{array}{l}\text { Condiciones ambientales } \\
\text { Exposición a temperaturas extremas, ruidos y vibraciones } \\
\text { Contacto con sustancias químicas } \\
\text { Duración de la jornada de trabajo } \\
\text { Pausas o descansos durante la jornada } \\
\mathrm{N}^{\circ} \text { de días de descanso semanal } \\
\text { Elementos de protección personal necesarios para el desempeño de la tarea, provisión de } \\
\text { elementos de protección personal por la empresa y uso } \\
\text { Provisión de agua } \\
\text { Cobertura de aseguradora de riesgos del trabajo } \\
\text { CAPACITACIÓN } \\
\text { ACtividades realizadas por la empresa } \\
\text { ACCIDENTES DE TRABAJO } \\
\text { ACcidentes laborales y muertes de compañeros del trabajador } \\
\text { Accidentes del trabajador (causas, tipo de lesión, asistencia de una aseguradora de riesgos del } \\
\text { trabajo, presencia de una lesión o discapacidad permanente) } \\
\text { VIOLENCIA EN EL TRABAJO } \\
\text { Situaciones de amenaza, presión o acoso }\end{array}$ \\
\hline SALUD Y OCUPACIÓN & $\begin{array}{l}\text { Percepción del estado de salud } \\
\text { Padecimientos o dolores derivados del trabajo } \\
\text { Tipo de padecimiento } \\
\text { Enfermedades laborales diagnosticadas por un médico. }\end{array}$ \\
\hline $\begin{array}{l}\text { ALOJAMIENTO PARA } \\
\text { TRABAJADORES MIGRANTES }\end{array}$ & $\begin{array}{l}\text { Tipo de alojamiento } \\
\text { Cantidad de personas alojadas } \\
\text { Disponibilidad de baño } \\
\text { Disponibilidad de agua para consumo personal }\end{array}$ \\
\hline
\end{tabular}

A los fines de este artículo, se procesó la información de las provincias de Misiones, Corrientes y Entre Ríos por ser, como ya se dijo, las principales productoras forestales. Del total de trabajadores agrarios que relevó la Encuesta, se trabajó con el universo de asalariados que declararon haber estado ocupados en actividades forestales en los últimos 12 meses ( $n=113$ trabajadores).

Además, se complementó con el análisis de entrevistas en profundidad realizadas a trabajadores forestales $(n=30)$ y a contratistas 
prestadores de servicios $(\mathrm{n}=8)$, en el período 2010-2014, ambos insertos en empresas de distinto tamaño y niveles tecnológicos. Se entrevistó a diferentes informantes clave, tales como personal de empresas, de sindicatos, profesionales del Instituto Nacional de Tecnología Agropecuaria e intermediarios, con el propósito de identificar y acceder a los trabajadores y contratistas. El trabajo de campo se realizó en campamentos forestales en la localidad de Ubajay, San José y en barrios periféricos de la ciudad de Concordia (Entre Ríos) así como en los hogares de residencia de los trabajadores (Eldorado y Mado-Colonia Delicia, provincia de Misiones). Las entrevistas tuvieron una duración aproximada de dos horas. La información se registró en formato digital y, para el procesamiento y análisis de los datos, se utilizó el software Atlas.ti. Las principales dimensiones utilizadas fueron: características sociodemográficas, tipo de trabajador, trayectoria laboral, migración, tarea, formas de intermediación, condiciones de trabajo y condiciones de vida. Del total de las 38 entrevistas, en este artículo se citan testimonios de cuatro trabajadores y tres contratistas.

Este estudio se ajusta a los criterios éticos elaborados por el Comité de Ética del Consejo Nacional de Investigaciones Científicas y Técnicas (CONICET) para las Ciencias Sociales y las Humanidades, Resolución 2857 del Ministerio de Educación, Ciencia y Tecnología. Los entrevistados participaron voluntariamente, luego de haberles aclarado la confidencialidad y el anonimato, así como los objetivos de la investigación. Los datos personales fueron protegidos mediante la identificación con números correlativos.

\section{RESULTADOS}

\section{Características de la mano obra}

Las ocupaciones temporales involucraban al $52 \%$ de los trabajadores, con una media en el año de casi 6 meses de ocupación. Los trabajadores que se insertaban todo el año (permanentes) tenían una edad promedio mayor (40 años) que los transitorios (34 años), con una desviación típica de 11,3. Entre los temporarios había casos de jóvenes menores de 16 años.

El empleo temporal es una de las caras principales de la precariedad laboral de los forestales, al carecer de estabilidad laboral y sufrir desocupación en ciertos períodos de tiempo. Asociado a esto, es mayor también la informalidad entre los trabajadores temporarios: la ausencia de registro del trabajador significa la carencia de beneficios sociales (aporte jubilatorio, cobertura de salud, vacaciones, días por enfermedad, etcétera). Esta situación afectaba al $63 \%$ de los temporarios, en tanto el $44 \%$ de los que estaban ocupados los 12 meses, eran informales. Como se puede observar, la permanencia anual en la ocupación no redunda necesariamente en una total formalización de la mano de obra, ya que buena parte de ellos no perciben los derechos sociales. La cobertura de una aseguradora de riesgos del trabajo (ART) alcanzaba a la mitad de los trabajadores, el 55\% de los cuales eran permanentes.

Asimismo, otra particularidad de estos sujetos era su baja escolaridad: más de la mitad no alcanzó el nivel mínimo de instrucción formal $(7,1 \%$ sin educación formal, $44,2 \%$ con el nivel primario incompleto); solo 3 de cada 10 completó el nivel primario $(30,1 \%)$ y, entre 1 y 2 de cada 10 , asistió al secundario, pero no lo concluyó; solo 3,6\% lo terminó.

\section{Riesgos por las condiciones del medio ambiente físico de trabajo}

La exposición a factores naturales, técnicos y organizativos adversos en el medio ambiente de trabajo, son también un riesgo para la salud del operario forestal ${ }^{(21)}$. Las condiciones meteorológicas desfavorables (viento, Iluvia y temperaturas extremas) constituyen un factor de riesgo de caída del trabajador cuando la superficie se encuentra embarrada, de aplastamiento por la caída de ramas en días de mucho viento y de deshidratación cuando están desarrollando la tarea con temperaturas muy elevadas. 
De la encuesta se desprende que el factor considerado más adverso por el trabajador son las altas temperaturas predominantes en el área en estudio. El 90\% de los encuestados manifestó estar expuesto a días de intenso calor.

El 50\% reportó estar expuesto a vibraciones, el $40 \%$ a niveles de ruido intensos (20,5\% debía hablar en voz alta; el $8 \%$ solo se podía comunicar gritando y el $11,5 \%$ no se podía comunicar) y el $49 \%$ a sustancias químicas. La pérdida de audición, los dolores de espalda y de la musculatura y el padecimiento conocido como "dedos blandos", son algunas de las enfermedades profesionales típicas de los operadores de motosierras; es por ello que se hace imprescindible el uso de elementos de seguridad, realizar descansos durante la jornada laboral, adoptar una buena postura al realizar el volteo, etcétera. En tanto son habituales los problemas respiratorios y de alergias entre aquellos que deben aplicar sustancias químicas a las plantaciones:

En Entre Ríos yo laburaba de noche, fumigando, por el viento. Pero lo dejé porque me enfermé. Me hacía mal el veneno, me daba taquicardia. Me daban el traje completo, las botas pero algo siempre nos queda. Yo no podía respirar, como si me faltara aire. Con la protección igual a mí me hace mal. Me asfixia. Para mí es prohibido pulverizar, me hace mal lo mismo. Fumigaba antes cuando era gurí, tenía 16, 17 años y no usábamos protector, nada, atropellábamos el capuerón, íbamos con mangueras, así nomas! (Trabajador 1, misionero, 32 años; entrevista realizada en su hogar en Mado, Misiones)

\section{Condiciones de trabajo}

La jornada laboral promedio era de 9 horas. Con respecto a los descansos o pausas en el trabajo, el $60 \%$ de los trabajadores las realizaba según sus propias necesidades, el $30 \%$ según lo establecido por el empleador y el $9 \%$ no hacía ninguna a lo largo de la jornada.
Con respecto a los descansos semanales, el $10 \%$ no disponía de ningún día, el $36 \%$ solo de un día y el $54 \%$ de más de un día.

Por otra parte, al $44 \%$ de los asalariados no le proveían el agua para el consumo durante el desempeño de la tarea, y el propio trabajador debía encargarse de ello. La distancia que debía recorrer dentro de la plantación, entre los distintos puestos de trabajo, hacía especialmente dificultoso el acceso al agua potable, sobre todo, en los días de mucho calor, cuando el propio obrero debía trasladar el agua que iba a consumir durante la jornada.

\section{Seguridad: elementos de protección personal}

En la Encuesta se preguntó acerca de qué elementos de protección personal el trabajador consideraba como necesarios en la práctica de la actividad forestal (incluyendo volteo-apeo, fumigación y carga). De estos, se relevó cuáles eran suministrados por el empleador $y$, a su vez, cuáles usaban en su tarea (Tabla 3).

En general, alrededor de 6 de cada 10 de todos los elementos de protección personal señalados como necesarios, eran suministrados por el empleador. En tanto el trabajador los utilizaba en una proporción mayor a la proporcionada por su patrón (mayor de 10 a 20 puntos relativos). Los encuestados no mencionaron el uso de indumentaria específica como pantalón anticortes, ni tampoco de vestimenta de colores fuertes que permiten la rápida visualización.

Por otra parte, el análisis de las entrevistas en profundidad a trabajadores, reveló que la utilización de una vestimenta adecuada (casco, borceguíes con puntera de acero, guantes, protector auditivo, visera facial y pantalones anticortes) solo era usada en forma total por los trabajadores de grandes empresas forestales o de contratistas que trabajan para estas y/o aserraderos de mayor envergadura. En el caso de contratistas más chicos, solo era frecuente el uso del casco si los trabajadores estaban cubiertos por una 
Tabla 3. Porcentaje de elementos de protección personal evaluados como necesarios por el trabajador, proporcionados por el empleador y usados en la tarea (n=113). Provincias de Misiones, Corrientes y Entre Ríos (Argentina), 2014.

$\begin{array}{lccc}\text { Elementos de protección personal } & \text { Necesarios } & \begin{array}{c}\text { Proporcionado } \\ \text { por empleador }\end{array} & \text { Usados } \\ \text { Guantes (anticortes o de látex para aplicación de agroquímicos) } & 65,5 & 66,2 & 79,7 \\ \text { Borceguíes/zapatos de seguridad } & 46,8 & 66,0 & 86,8 \\ \text { Casco } & 38,9 & 65,9 & 72,7 \\ \text { Botas de goma } & 38,9 & 63,6 & 86,4 \\ \text { Antiparras (protección de ojos) } & 33,6 & 65,8 & 65,8 \\ \text { Mascarilla (protección de sustancias químicas, polvillo) } & 30,1 & 55,9 & 64,7 \\ \text { Traje impermeable } & 23,9 & 63,0 & 77,8 \\ \text { Faja lumbar } & 22,1 & 60,0 & 76,0 \\ & & & \\ \text { Fuente: Elaboración propia a partir de la información de la Encuesta sobre Empleo, Protección Social y Condiciones de Trabajo } \\ \text { de los Asalariados Agrarios }\end{array}$

aseguradora de riesgos del trabajo. Cabe aclarar que, de lo expresado tanto por los contratistas como por los mismos trabajadores, se desprende la reticencia de estos últimos para incorporar las medidas de seguridad. La resistencia al uso de los elementos de protección personal se debe a que pueden generarle algunas incomodidades o molestias, por lo que es fundamental que los trabajadores perciban cuáles son los riesgos que involucran las distintas tareas. La capacitación de trabajadores y contratistas en el uso de un equipamiento de seguridad es central para generar, en ambos, una conciencia del riesgo: en los primeros, para que lo incorporen en su rutina y, en los segundos, para que se los provean. El entrenamiento en las normas de seguridad por parte del empleador así como la supervisión en el lugar de trabajo forman parte del proceso de consolidación del hábito de incorporación de los elementos de protección personal por el trabajador y de su familiarización, al internalizar los riesgos específicos de la tarea:

El casco, por ejemplo, mi personal no estaba acostumbrado a usarlo; lo implantamos y ahora todo el mundo está con el casco. Y llega al lugar de trabajo y es lo primero que hace. Antes tenía que estar dos veces por día diciendo "usalo, por favor. No podés trabajar sin el casco"; hemos tenido hasta discusiones con el personal; decirle "bueno, te vas si no lo usás". Y se acostumbró. Hace unos días un empleado se lo olvidó y le fue a decir al encargado "no puedo trabajar porque no tengo el casco". Están concientizados, pero nos lleva mucho tiempo. (Contratista 1, entrerriano, 45 años de edad, con una antigüedad de 15 años como contratista forestal; entrevista realizada en un campamento cercano a la localidad de Ubajay)

Uno a veces dice "yo no voy a usar casco..." pero ¡locos somos nosotros! Si realmente estás en negro no te dan nada, no te dan casco, borceguíes, protector, nada. Si tenés un accidente ¿quién te paga? Si te mata una madera, tu familia ¿Cómo vive? Si uno está en blanco entonces tu familia tiene cómo seguir. Esta clase de trabajo es en la que hay más accidentes. (Trabajador 2, paraguayo, 29 años; entrevista realizada en su hogar en la localidad de Mado).

Usamos borceguíes con punta de acero por dentro y si te pegás un hachazo te bandea. Antes siempre íbamos de zapatillas por más fresco y siempre nos cortábamos. Hace como seis años que 
empezamos a usar este calzado. (Trabajador 3, misionero, de la localidad de Bernardo de Irigoyen, 35 años; entrevista realizada en un campamento próximo a la ciudad de Concordia).

\section{Formación de la mano de obra}

El aprendizaje en el desempeño de la tarea es lo habitual en esta mano de obra que, además, evidencia niveles bajos de escolarización. Esta situación se ve reflejada cuando se observa que solo el $31 \%$ de los trabajadores declaró haber recibido capacitación en las empresas donde se desempeñaba. El $24 \%$ de estas fue en normas de seguridad e higiene, el $20 \%$ en técnicas vinculadas a la tarea, el $17 \%$ en primeros auxilios, el $16 \%$ en manejo del fuego, el $10 \%$ en el uso de maquinarias y herramientas y el $13 \%$ restante en otros temas.

Una cuestión que surge de las entrevistas a trabajadores es la valorización positiva del aprendizaje de la tarea entre familiares y pares desde temprana edad, así como haber sido socializados en un entorno geográfico en donde predomina la actividad del monte. Las nociones de aptitud para realizar un trabajo pesado, el conocimiento de la tarea y la alta productividad en el desempeño están profundamente internalizadas entre los trabajadores entrevistados, esto contribuye a que subestimen la necesidad de instancias de capacitación.

\section{Otros factores de riesgo}

Los riesgos a los que está expuesta la mano de obra forestal no se limitan a las condiciones del medio ambiente del trabajo, a las faltas de medidas de seguridad de protección personal, sino también a cuestiones psicosociales del entorno laboral, relacionadas con el contenido y la organización del proceso de trabajo. Estos riesgos alteran los aspectos cognitivos y psíquicos del trabajador; el sufrimiento generado puede repercutir en la salud, provocando somatizaciones e incrementando las posibilidades de padecer lesiones, accidentes o enfermedades como consecuencia del estrés ${ }^{(13)}$.
De la información de la Encuesta se desprende que las presiones ejercidas por los empleadores son las predominantes entre los forestales. El 12\% de los trabajadores reportó haber estado sujeto a situaciones de desvalorización profesional o personal, así como a maltrato verbal; el $12 \%$ debió soportar presión/agresión de parte de sus jefes; el 10\% recibió amenazas de despido y al $9 \%$ se le aplicaron restricciones para comunicarse con sus compañeros.

\section{Accidentes laborales}

En el último año, el $17 \%$ de los encuestados declaró haber sufrido un accidente en el desempeño del trabajo. Los siniestros fueron producidos principalmente por el contacto con objetos cortantes y punzantes (53\%), la caída de objetos (ramas, herramientas u otros materiales, $26 \%$ ), la caída del trabajador $(21 \%)$ o el esfuerzo por manipulación manual de cargas $(16 \%)$. Estas causas de accidentes fueron señaladas también como las más frecuentes por otros estudios realizados en la Argentina y en otros países ${ }^{(22,23)}$

Además, el $25 \%$ reportó que alguno de sus compañeros había tenido un accidente realizando su trabajo (en los últimos 12 meses); el $4 \%$ de estos ocasionaron la muerte del trabajador:

\footnotetext{
Mayormente sucede el accidente por falta de pensar o porque tiene pensamientos, piensa por la familia o quizás tiene otro problema. Pueden venir de muchos problemas los accidentes, porque muchos trabajadores trabajan..., no somos todos iguales. Yo en cambio, cuando empiezo a trabajar, yo me concentro en mi trabajo; yo dejo otros problemas para atrás. Tengo que estar concentrado en el trabajo para no accidentarme; tengo que cuidarme porque es un trabajo muy peligroso el que nosotros hacemos. Todos saben que es peligroso, pero como le digo, que muchos pensamos diferente. Muchos tienen problemas familiares, muchos problemas; entonces piensan en eso y
} 
sin darse cuenta, se accidentan... Puede pasar. O tenés una tarea que tenés que terminar y tenés que... Ahí puede ocasionar accidentes. Muchas horas, trabajás muchas horas. Porque hay lugares que vos tenés que trabajar muchas horas para que vos tengas una ganancia, ¿no es cierto? (Trabajador 4, misionero, 55 años; entrevista realizada en su hogar, en un barrio de la ciudad de Eldorado)

Los accidentes se relacionan con el uso de determinadas herramientas. Las que más se utilizaron fueron: motosierra $(40 \%)$, machete $(32 \%)$, hacha $(13 \%)$, mochila pulverizadora $(12 \%)$, serrucho $(6 \%)$ y tractor $(12 \%)$. La motosierra a cadena es la más peligrosa de todas las usadas en la actividad y, además, su operador es el más expuesto a enfermedades profesionales. Se recomienda, como medida preventiva, el buen mantenimiento de las herramientas y usar siempre los equipos de protección individual. La verificación del correcto estado de la cadena (engrasada, tensada y afilada en forma adecuada), el uso de los elementos de protección personal que requiere la tarea, así como el modo en que se debe realizar la práctica (mantener ambas manos en la motosierra cuando se está cortando, desplazarse con el motor apagado, etcétera) son algunos de los protocolos a seguir para evitar los riesgos ${ }^{(23)}$ :

Se han presentado accidentes, sí. Cortes generalmente con la motosierra, en piernas, por el rebote de las máquinas. Todo por falta de capacitación del operario o por demasiada confianza. Porque acá hay dos cosas, se le exige y se le exige al empleador que tiene que tener las cosas, pero el empleado no es que cumple por voluntad propia, continuamente tiene que tener un supervisor que les esté marcando las cosas. (Contratista 2, uruguayo, 33 años de edad, con una antigüedad de 6 años como contratista forestal en la zona del NE de Entre Ríos y SE de Corrientes; entrevista realizada en un campo forestal próximo a Colonia Berduc, Entre Ríos).
La bibliografía da cuenta de la importancia que tiene la utilización de métodos adecuados para el corte de los árboles -la tarea más riesgosa de la actividad forestal- en la reducción de la tasa de siniestralidad. El volteo dirigido consiste en orientar la caída del árbol hacia zonas abiertas, considerando las características del ejemplar, la topografía y la dirección del viento. Una vez realizado el corte, las ramas o árboles suspendidos en otras plantas son la causa de los accidentes de mayor peligrosidad y que ocasionan la mayor cantidad de muertes.

De las entrevistas en profundidad a contratistas, varios testimonios expresan la necesidad de realizar un buen planeamiento y organización del volteo para evitar riesgos. Tanto trabajadores como contratistas hicieron mención de accidentes ocurridos por la presencia de ramas o plantas que quedaron colgadas de otras, y que luego cayeron y provocaron accidentes de trabajadores.

Uno de los contratistas observó la necesidad de establecer un espacio de separación entre el área de operación de dos motosierristas, delimitado por 3 o 4 luchas (hileras de árboles). El volteo afecta también a los que están trabajando alrededor de este, como es el caso del asistente, encargado de apear, acomodar las maderas y descortezar (en el caso del eucalipto). El asistente está expuesto a la caída de ramas, así como al aplastamiento y a padecer trastornos en la columna, ocasionados por el gran esfuerzo físico que involucra el arrastre y apilado de la madera.

\section{Salud, enfermedades laborales y patologías}

La mayor parte de los trabajadores consideró que su estado de salud era bueno ( $57 \%$ ), mientras que el $29 \%$ evaluaba que era regular, el $12 \%$ muy bueno y menos del $2 \%$ malo. Del total de trabajadores encuestados, cerca del $10 \%$ declaró haber tenido, durante los últimos 12 meses, una enfermedad causada por el trabajo y diagnosticada por un médico.

Sin embargo, al indagar sobre el padecimiento de alguna patología sufrida mientras se encontraba ocupado en esta actividad, los 
porcentajes aumentan notablemente. El $60 \%$ manifestó haber sufrido cansancio y falta de energía, el $46 \%$ dolor de cabeza y alrededor del $15 \%$ alteraciones en el sueño, en la vista y mareos (Tabla 4).

\section{DISCUSIÓN}

Los resultados presentados evidencian las coincidencias con la extensa bibliografía sobre las condiciones en las que se desempeña el trabajador forestal y los riesgos a los que está expuesto. Por las características y las demandas de la actividad (exigencia física, alta productividad laboral y residencia en campamentos aislados, entre otras) involucra a trabajadores jóvenes, con escasa escolarización, con problemas de empleabilidad y precariedad laboral| ${ }^{(24,25,26)}$.

En forma similar a lo señalado por otros autores para el caso de EEUU, donde se apela a la mano de obra latina procedente de México y de otros países de América Cen$\operatorname{tral}^{(27,28,29)}$, la fuerza de trabajo en el área estudiada tiene una alta movilidad territorial; por ejemplo, los misioneros suelen ocuparse en distintas plantaciones del interior de su provincia, migrando temporariamente a los montes implantados de Corrientes y de Entre $\operatorname{Ríos}^{(30)}$. La informalidad y la precarización laboral de la actividad son rasgos comunes en todo el mundo ${ }^{(5,23,31,32)}$.

Asimismo, los hallazgos están en sintonía con lo que se analiza en otros estudios sobre esta temática, en los que se señalan las altas tasas de lesiones, enfermedades laborales y letalidad de la fuerza de trabajo en regiones productoras del nordeste y el sudeste de EEUU ${ }^{(33,34)}$. La tasa de accidentes $(17 \%)$ es semejante a la observada en otras investigaciones $(16,3 \%)^{(35)}$. Tanto en la Encuesta como en las entrevistas, los trabajadores manifestaron haber sufrido, en el último año, cortes y heridas causados por el uso de motosierras, hachas y otras herramientas. Como expresan otros autores respecto de la situación de los trabajadores en Brasil, esta mano de obra se ve sometida a una alta exigencia física
Tabla 4. Patologías o síntomas de enfermedades padecidas durante el trabajo, por los trabajadores forestales ( $n=113)$. Provincias de Misiones, Corrientes y Entre Ríos, (Argentina), 2014.

Tipo de patología o síntomas

Cansancio o falta de energía $\quad 60,2$

Dolor de cabeza

46,0

Alteraciones del sueño

15,0

Alteraciones de la vista

15,9

Mareos

15,0

Inflamación de pies, piernas, manos

10,6

Alteraciones en los huesos (artrosis, artritis, etc.) $\quad 10,6$

Humor irritable y tensión nerviosa

10,6

Reducción de la audición

9,7

Hipertensión

8,8

Alteraciones cardiológicas

8,0

Dificultades para mantener la atención

8,0

Alteraciones respiratorias (asma, broncoespasmos, alergias) $\quad 7,1$

Alteraciones en la piel (urticaria, picazón, irritación, etc.)

7,1

Fuente: Elaboración propia a partir de la información de la Encuesta sobre Empleo, Protección Social y Condiciones de Trabajo de los Asalariados Agrarios ${ }^{(20)}$.

en función de alcanzar una mayor productividad (remuneración por rendimiento) y a presiones psicológicas en virtud de la inestabilidad del tipo de empleo y de la alta rotación del personal contratado ${ }^{(36)}$.

Además de ser una ocupación riesgosa, demanda una fuerte exigencia física, con exposición a condiciones ambientales adversas como vibraciones y ruido. Con el paso de los años en la actividad, los trabajadores evidencian un deterioro corporal provocado por la intensidad de las tareas ${ }^{(37,38)}$; la incidencia de enfermedades profesionales como pérdidas de audición por la exposición al ruido, dolor muscular y lesiones en la columna son algunos de los resultados relevantes del estudio realizado.

El bajo nivel de escolaridad es un indicador adverso a tener en cuenta para el desarrollo de actividades de capacitación, sobre todo, para el entrenamiento en el uso de maquinarias. El saber hacer, adquirido básicamente con la experiencia en el trabajo, la falta de capacitación y la carencia de información acerca de los riesgos redundan en la reiteración de prácticas indebidas, nocivas 
para la salud y la calidad de vida del trabajador. A su vez, la subestimación de los riesgos por parte del asalariado puede deberse a la necesidad de minimizar las experiencias negativas como mecanismo defensivo y/o a sobreestimar su propia capacidad para ejercer un control sobre los peligros del proceso de trabajo. La naturalización del riesgo como parte del trabajo se debe, muchas veces, a que la mano de obra lo percibe como una exigencia laboral más entre otras. Esta percepción se construye sobre la base de prácticas en el lugar de trabajo que combinan las experiencias propias y las compartidas con otros compañeros ${ }^{(39)}$.

En la forestación -como en la minería y en la construcción- al ser una actividad ejercida exclusivamente por hombres, suele observarse en el colectivo una necesidad de exaltar ciertos atributos asociados a la masculinidad, como son el coraje, la valentía, la falta de miedo y la fortaleza para resistir las condiciones adversas ${ }^{(40)}$. Es por ello que se requiere de análisis que profundicen e indaguen acerca de las dimensiones que puedan ayudar a acrecentar la familiaridad de comportamientos seguros en las cuadrillas de tala de árboles ${ }^{(34)}$. La incorporación de hábitos y prácticas seguras a través de instancias de capacitación que sirvan para concientizar al trabajador acerca de los riesgos, es un tema recurrente en los estudios sobre sus condiciones laborales:

Es tanta la confianza que llega a cazar al oficio que ahí es donde viene el descuido. Cuando está aprendiendo el oficio probablemente tenga menos riesgos de accidentes o más riesgos, pero con más cuidado creo. Y después, a la vez que se van poniendo duchos, y bueno la confianza lleva por ahí al accidente. (Contratista 3, entrerriano, 58 años de edad, con una antigüedad de 35 años como contratista forestal; entrevista realizada en un campamento cercano a la ciudad de Concordia).

El efectivo cumplimiento de las normas y medidas de seguridad orientadas a prevenir lesiones y accidentes depende de la voluntad y el compromiso de las empresas en la gestión y capacitación de los recursos humanos, así como del control que ejerzan de los contratistas encargados del suministro de la mano de obra (provisión de elementos de protección personal, supervisión del uso correcto en el lugar de trabajo, abastecimiento de agua potable, alojamientos dignos, registro de los trabajadores, etcétera). Es fundamental, además, el papel del Estado en la elaboración de políticas públicas destinadas a regular el trabajo en la forestación y como agente de control del cumplimiento de las normas para que se generen empleos dignos ${ }^{(31)}$.

\section{CONCLUSIONES}

La combinación de una metodología cuanticualitativa basada en la Encuesta sobre Empleo, Protección Social y Condiciones de Trabajo de los Asalariados Agrarios y en entrevistas en profundidad, complejiza y enriquece el análisis de las condiciones laborales de los obreros forestales. Este tipo de enfoque responde a la necesidad de abordar distintos aspectos del fenómeno analizado, complementando la información estadística con la perspectiva de los propios actores.

Este estudio pone de manifiesto el alcance que asume el proceso de trabajo y las condiciones del medio ambiente y los factores psicosociales en la forestación, y el alto impacto que tienen en la salud del trabajador, en el desarrollo de enfermedades ocupacionales y en la presencia de accidentes. Esto se sustenta, también, en otros factores externos al mundo del trabajo, pero que actúan como condicionantes de este, principalmente las diferencias socioeconómicas y culturales en la estratificación social más amplia.

Es importante considerar ciertas cuestiones que se vinculan con la socialización desde temprana edad en la vida y el trabajo en el monte, como es el caso de buena parte de la mano de obra de origen misionero y la cultura del propio trabajo forestal. Ambas generan identidades específicas que, muchas 
veces, conducen a la minimización del riesgo o a conferir un exceso de confianza por la cotidianeidad de las prácticas que lleva a correr riesgos $^{(13,19)}$. A su vez, aspectos vinculados a la masculinidad juegan un papel a tener en cuenta en el comportamiento de los forestales y en la construcción que ellos tienen del riesgo ${ }^{(14,40)}$.

Se puede pensar en la existencia de una cierta inmunidad subjetiva ${ }^{(15)}$, en el sentido de que esta confianza los conduce a subestimar los peligros que conllevan diariamente las tareas, desde los menos graves, a los más peligrosos como puede ser dejar enganchadas ramas cuando se realiza el volteo. Como contracara, estas situaciones generan un valor de culpabilización hacia los sujetos que sufren accidentes, tanto de los empleadores como, llamativamente, de sus compañeros.

Se pudo observar que la implementación de las normas sobre las condiciones de seguridad, en el espacio concreto del trabajo, insume tiempo, y que se van construyendo acuerdos entre las partes para su incorporación. La marcada precariedad de las inserciones, la transitoriedad de la ocupación, así como formas de remuneración ancladas en el destajo, generan un sistema que fragmenta el efectivo acceso a mejores condiciones laborales. Por su parte, la difusión de la tercerización y la rotación de los trabajadores entre distintos contratistas, impulsa a contracorriente los beneficios de su adopción para el trabajador. Muchas veces el intermediario, por designio de la empresa, es el encargado de entregar elementos de protección, sin proveer al trabajador la información necesaria y las ventajas de su uso, generando en el grupo de trabajadores más una carga que un beneficio. Otro elemento que se destaca es la traba que produce desarrollar la actividad en un ambiente adverso (altas temperaturas), lo cual redunda en ciertas resistencias por parte de los trabajadores a incorporar indumentarias que potencian las temperaturas adversas en las que se realizan las tareas.

El trabajo informal, el saber hacer basado en la experiencia y la baja incidencia de instancias de capacitación constituyen elementos de mayor vulnerabilidad y riesgo frente a las buenas prácticas laborales.

Aunque la información brindada a los trabajadores en temas de seguridad y de prevención no garantiza la efectividad de la percepción del riesgo, de los testimonios tanto de contratistas como de trabajadores, se desprende que, una vez sorteado un tiempo de adaptación al uso de los elementos de protección, se supera la incomodidad que producía en sus inicios, se adquiere el hábito y, luego, es el trabajador el que demanda y reclama su provisión.

Si bien los asalariados manifiestan mejoras en las condiciones laborales en los últimos años, todavía se registra una alta tasa de accidentes. Aunque existe una mayor conciencia de la necesidad del uso de elementos de protección personal, aún no se encuentra generalizado. El suministro por parte del empleador es más bajo que el uso efectivo que hacen de esos elementos los trabajadores. A su vez, una extensa jornada de trabajo para realizar una tarea que exige un alto grado de concentración tiende a favorecer la probabilidad de ocurrencia de un accidente.

Por último, es fundamental que los responsables en la generación de normativas que favorezcan las buenas condiciones laborales conjuguen los desarrollos técnicos en función de cómo se organiza y estructura la mano de obra en un mercado y en un proceso de trabajo específico (prácticas laborales y mundo de vida de los trabajadores). Es relevante destacar que algunas situaciones de mayor exposición al riesgo, responden más al modelo organizativo del proceso de trabajo (cantidad de horas, intensidad, repeticiones de la tarea, etcétera), que a la ausencia de medidas preventivas y al uso de elementos de protección personal, lo que no significa quitarle importancia a estos últimos.

En síntesis, las atribuciones que definen los conceptos de salud, riesgo y enfermedad se encuentran determinadas por las condiciones sociales y culturales en las que se encuentran insertas. El entorno social, las cuestiones de desigualdad en la estratificación social y las diferencias de poder que están implícitas interactúan en la construcción de la idea de salud y riesgo laboral. Por un lado, el sector 
empresarial concibe ese bienestar en función del puesto del trabajador $y$, por el otro, el grupo de trabajadores corre con desventajas sociales (por la condición migratoria, el escaso acceso a la educación y a la salud, ingresos bajos, entre otras) y que percibe su condición de empleabilidad como la posibilidad de acceso a un empleo, a costas de "naturalizar" las condiciones en las que se desenvuelve.

\section{AGRADECIMIENTOS}

Las autoras agradecen los comentarios de los evaluadores externos y de los editores de la revista. Este artículo se realizó, en forma parcial, con información recopilada en una investigación más amplia, en el marco del proyecto "El trabajo migrante transitorio en la Argentina", dirigido por Guillermo Neiman, y financiado por la Agencia Nacional de Promoción Científica y Tecnológica (Ministerio de Ciencia, Tecnología), en el Centro de Estudio e Investigaciones Laborales (CEIL-CONICET), PICT 232/07, finalizado en el año 2014.

\section{REFERENCIAS BIBLIOGRÁFICAS}

1. Organización Internacional del Trabajo. Cuando el trabajo lesiona: rayos $\mathrm{X}$ a la seguridad laboral. Trabajo [Internet]. 1997;(21) [citado 1 ago 2017]. Disponible en: https://tinyurl.com/d6kxy7e.

2. Ministerio de Agricultura, Ganadería y Pesca, Unidad para el Cambio Rural, Presidencia de la Nación. Argentina: Plantaciones Forestales y Gestión Sostenible, 2014 [citado 26 dic 2016]. Disponible en: https://tinyurl.com/ycwk39wf.

3. Subsecretaría de Agricultura, Dirección de Producción Forestal. Sector forestal: año 2012 [Internet]. Buenos Aires: Ministerio de Agricultura, Ganadería y Pesca; 2013 [citado 26 dic 2016]. Disponible en: https://tinyurl.com/y7cylsj3.

4. Pinto Leite A. Análise da terceirização na colheita florestal no Brasil. [Teses de Doutorado]. Minas Gerais: Universidade Federal de Viçosa; 2002.

5. Mann G. The state, race and "wage slavery" in the forest sector of the Pacific Northwest United States. The Journal of Peasant Studies. 2001;29(1):61-88.
6. Pucci F, Nión S, Ciapessoni F. Condiciones de trabajo y gestión del riesgo en las empresas forestales uruguayas [Internet]. Montevideo: Universidad de la República Uruguay; 2012 [citado 24 jul 2017]. Disponible en: https://tinyurl.com/ycjfxew7.

7. Alberto M, Iñiguez MJI, Marensi P. Prediagnóstico sobre las condiciones y medio ambiente del trabajo en la actividad de la forestación en la Mesopotamia [Internet]. Buenos Aires: Ministerio de Trabajo, Empleo y Seguridad Social; Superintendencia de Riesgos del Trabajo; 2007 [citado 24 dic 2016]. Disponible en: https://tinyurl.com/ y8syrza7.

8. Bardomás S, Díaz D. Trabajadores migrantes temporarios en la actividad forestal de la provincia de Entre Ríos. En: Benencia R, Pedreño Cánovas A, Quaranta G, (comps.). Mercados de trabajo, instituciones y trayectorias en distintos escenarios migratorios. Buenos Aires: Ciccus; 2014. p. 139-162.

9. Poshen P. Industria forestal [Internet]. En: Poshen P, (dir.). Enciclopedia de Salud y Seguridad del Trabajo. No. 68. Ginebra: Organización Internacional del Trabajo; 1998 [citado 9 ene 2016]. Disponible en: https://tinyurl.com/ycrmrx74.

10. Badía Montalvo R. Salud ocupacional y riesgos laborales. Boletín de la Oficina Sanitaria Panamericana. 1985;98(1):20-33.

11. Kornblit AL, Mendes Diz AM. La salud y la enfermedad: aspectos biológicos y sociales. Buenos Aires: Aique; 2000.

12. Menéndez E. El modelo médico y la salud de los trabajadores. Salud Colectiva. 2005;1(1):9-32.

13. Neffa JC. Los riesgos psicosociales en el trabajo: contribución a su estudio. Buenos Aires: CEILCONICET, Universidad Nacional del Nordeste, Universidad Nacional de La Plata, Universidad Nacional de Moreno, Universidad Metropolitana para la Educación y el Trabajo, Centro de Innovación para los Trabajadores; 2016. 
14. Del Águila A. "El que no se la banca, mejor que se dedique a otra cosa": riesgo, masculinidad y clase social entre trabajadores paraguayos en la industria de la construcción del AMBA. Runa. 2015;36(1):51-72.

15. Douglas M. La aceptabilidad del riesgo según las ciencias sociales. Barcelona: Paidós; 1996.

16. Urteaga $E$, Eizagirre A. La construcción social del riesgo. EMPIRIA-Revista de Metodología de las Ciencias Sociales. 2013;25:147-170.

17. Cobos Sanchiz D, Pérez Pérez I, Reyes Costales E. La visión sobre los riesgos laborales y la cultura preventiva de los estudiantes universitarios: un estudio exploratorio en la Universidad Pablo de Olavide. Bordón. 2011;63(3):75-90.

18. Neffa JC. ¿Qué son las condiciones y medio ambiente de trabajo?: Propuesta de una nueva perspectiva. Buenos Aires: Área de Estudio e Investigación en Ciencias Sociales del Trabajo, Centro de Estudios e Investigaciones Laborales, CREDAL-CNRS, Humanitas; 1989.

19. Martínez Guirao J. Riesgos laborales en la construcción: un análisis sociocultural. Universitas, Revista de Ciencias Sociales y Humanas de la Universidad Politécnica Salesiana del Ecuador. 2015;(23):65-86.

20. Ministerio de Trabajo, Empleo y Seguridad Social de la Nación; Registro Nacional de Trabajadores y Empleadores Agrarios; Superintendencia de Riesgos del Trabajo. Encuesta sobre Empleo, Protección Social y Condiciones de Trabajo de los Asalariados Agrarios. Buenos Aires: MTEySS, Renatre, SRT; 2016.

21. Belanová K, Vlcková M, Olajec I. Working place conditions impact on work injury occurrence in the timber harvesting process. Human Resources Management \& Ergonomics. 2010;4(2):1-12.

22. Superintendencia de Riesgos del Trabajo. Accidentabilidad laboral en sectores específicos de la economía, año 2012 [Internet]. Buenos Aires: Unidad de Estudios Estadísticos, GPIEyCG, Superintendencia de Riesgos del Trabajo; 2014 [citado 26 jul 2017]. Disponible en: https://tinyurl.com/ yau8zrw6.

23. Ciudad-Valls I. Prevención de riesgos laborales en trabajos forestales. Seguridad y Salud en el Trabajo. 2013;(71):12-19.

24. Peirano $C$, Bustos Hinostroza $M$, Nahirñac $P$. Recursos humanos en el sector forestal: un análisis cuantitativo y cualitativo a partir del programa de certificación de competencias laborales [Internet]. En: XIII Congreso Forestal Mundial; Buenos Aires; 2009 [citado 26 jul 2017]. Disponible en: https:// tinyurl.com/y7efwm72.

25. Bardomás S. Calidad de vida y condiciones laborales en la actividad forestal en la Argentina. En: Tolón Becerra A, Lastra Bravo X, (eds.). Actas del I Seminario Internacional de Cooperación y Desarrollo en Espacios Rurales Iberoamericanos: sostenibilidad e indicadores. Almería: Universidad de Almería; 2007. p. 461-473.

26. Carámbula Pareja M. Indicadores de sostenibilidad social en los territorios forestales: aportes para la construcción de indicadores de precariedad laboral. En: Tolón Becerra A, Lastra Bravo $X$, editores. Actas del I Seminario Internacional de Cooperación y Desarrollo en Espacios Rurales Iberoamericanos: sostenibilidad e indicadores. Almería: Universidad de Almería; 2007. p. 475-478.

27. McDaniel J, Casanova V. Pines in lines: tree planting, HsB guest workers, and rural poverty in Alabama. Southern Rural Sociology. 2003;19(1):73-96.

28. Casanova V, McDaniel J. "No sobra y no falta": recruitment networks and guest workers in southeastern U. S. forest industries. Urban Anthropology. 2005;34(1):45-84.

29. Sarathy B, Casanova V. Guest workers or unauthorized immigrants?: the case of forest workers in the United States. Policy Sciences. 2008;41:95114.

30. Bardomás SM. Trabajadores de aquí y de allá: la migración a dos mercados de trabajo agrícola de la Argentina. Estudios del Trabajo-ASET. 2009;(3738):55-84.

31. Pignati WA, Machado JMH, Mesquita, J. Riscos e agravos a vida dos trabalhadores das indústrias madeireiras de Mato Grosso. Ciencia \& Saúde Coletiva. 2005;10(4):961-973.

32. Estruch E, Rapone C, FAO. La promoción del empleo decente en el sector forestal para mejorar la nutrición y la seguridad alimentaria; documento de antecedentes para la conferencia internacional sobre los bosques para la seguridad alimentaria y nutricional [Internet]. Roma: FAO; 2013 [citado 25 jul 2017]. Disponible en: https://tinyurl.com/ yamlql8n.

33. Wilmsen C, Bush D, Barton-Antonio D. Working in the shadows: safety and health in forestry services in Southern Oregon. Journal of Forestry. $2015 ; 113(3): 315-324$. 
34. Conway SH, Pompeii LA, Casanova V, Douphrate DI. A qualitative assessment of safe work practices in logging in the Southern United States. American Journal of Industrial Medicine. 2017;60(1):58-68.

35. Canto Lorensi Do J, Machado Cardoso C, Souza De P, Garlet A, Carvalho Armond R, Noce R. Avaliação das condições de segurança do trabalho na colheita e transporte florestal em propiedades rurais fomentadas no Estado do Espírito Santo. Revista Árvore. 2007;31(3):513-520.

36. Pinto Leite A, Soares T, Saraiva Nogueira G, Vignote Peña S. Perfile qualidade de vida de trabalhadores de colheita florestal. Revista Árvore. 2012;36(1):161-168.

37. Blombäck P, Poschen P. ¿Es digno el trabajo del sector forestal?: mejora del trabajo forestal y de los medios de subsistencia que dependen de los bosques. Quebec: XII World Forestry Congress; 2003.

38. Riella A, Mascheroni P. Explorando la calidad del empleo en la forestación. Montevideo: Universidad de la República Uruguay; 2009.

39. Camarena Ojinaga L; Von Glascoe C, Martínez Valdés C, Arellano García E. Riesgos del trabajo y salud: percepción de mujeres indígenas jornaleras en el noroeste de México. Salud Colectiva. 2013; 9(2):247-256.

40. Alberti AV. La fuerza masculina y la fuerza animal: construcciones identitarias en torno a la migración laboral de trabajadores forestales en Argentina. Revista Chilena de Antropología. 2013;(28):29-51. 